\title{
MicroRNA-128-3p regulates mitomycin C-induced DNA damage response in lung cancer cells through repressing SPTAN1
}

\author{
Rui Zhang ${ }^{1,2, *}$, Chang Liu ${ }^{1,2, *}$, Yahan Niu ${ }^{1,2, *}$, Ying Jing ${ }^{1,2, *}$, Haiyang Zhang ${ }^{1,2}$, Jin \\ Wang $^{1,2}$, Jie Yang ${ }^{1}$, Ke Zen ${ }^{1,2}$, Junfeng Zhang ${ }^{1,2}$, Chen-Yu Zhang ${ }^{1,2}$ and Donghai $\mathrm{Li}^{1,2}$ \\ ${ }^{1}$ State Key Laboratory of Pharmaceutical Biotechnology, Nanjing Advanced Institute for Life Sciences(NAILS), School of Life \\ Sciences, Nanjing University, Nanjing, Jiangsu 210093, China \\ ${ }^{2}$ Jiangsu Engineering Research Center for microRNA Biology and Biotechnology, School of Life Sciences, Nanjing University, \\ Nanjing, Jiangsu 210093, China \\ *These authors contributed equally to this work
}

Correspondence to: Donghai Li, email: donghaili@nju.edu.cn Chen-Yu Zhang, email: cyzhang@nju.edu.cn Junfeng Zhang, email: jfzhang@nju.edu.cn Ke Zen, email: kzen@nju.edu.cn

Keywords: lung cancer, MicroRNA, mitomycin C, spectrin, DNA repair

Received: April 21, $2015 \quad$ Accepted: September 20, 2016

Published: September 28, 2016

Copyright: Zhang et al. This is an open-access article distributed under the terms of the Creative Commons Attribution License 3.0 (CC BY 3.0), which permits unrestricted use, distribution, and reproduction in any medium, provided the original author and source are credited.

\section{ABSTRACT}

The DNA damage response is critical for maintaining genome integrity and preventing damage to DNA due to endogenous and exogenous insults. Mitomycin C (MMC), a potent DNA cross-linker, is used as a chemotherapeutic agent because it causes DNA inter-strand cross-links (DNA ICLs) in cancer cells. While many microRNAs, which may serve as oncogenes or tumor suppressors, are grossly dysregulated in human cancers, little is known about their roles in MMC-treated lung cancer. Here, we report that miR-128-3p can attenuate repair of DNA ICLs by targeting SPTAN1 (aII Sp), resulting in cell cycle arrest and promoting chromosomal aberrations in lung cancer cells treated with MMC. Using computational prediction and experimental validation, SPTAN1 was found to be a conserved target of miR-128-3p. We then found that miR-128-3p caused translational inhibition of SPTAN1, reducing its protein level. SPTAN1 repression via miR-128-3p also induced cell cycle arrest and chromosomal instability. Additionally, miR-128-3p significantly influenced interaction of the aII Sp/ FANCA/XPF complex, thus limiting DNA repair. In summary, the results demonstrate that miR-128-3p accelerates cell cycle arrest and chromosomal instability in MMCtreated lung cancer cells by suppressing SPTAN1, and these findings could be applied for adjuvant chemotherapy of lung cancer.

\section{INTRODUCTION}

Mitomycin C (MMC) is an anti-tumor drug widely used in clinical cancer chemotherapy [1]. Use of MMC has been associated with a response rate of $20 \%$ in patients with non-small cell lung cancer (NSCLC), which is the most common malignant disease worldwide. Combination of MMC with ifosfamide in a previous phase 2 study of NSCLC achieved an overall response rate to chemotherapy of $43 \%$ [2]. Monofunctional and bifunctional alkylation of DNA by MMC has been proven to produce a single covalent DNA adduct and DNA inter-strand cross-links (DNA ICLs) [3], and these DNA pathological changes constitute the molecular basis of the cytotoxicity of MMC toward tumor cells. A later study reported that its cytotoxicities and derivatives are closely related to the observed high frequencies of DNA ICLs [4]. However, the molecular mechanism by which $\mathrm{MMC}$, as one of 3 most active agents, functions with microRNAs (miRNAs) to treat NSCLC patients remains unclear.

MiRNAs are a class of endogenous non-coding RNAs, typically 22 nucleotides in length, that function primarily by targeting the $3^{\prime}$-untranslated region ( $3^{\prime}$-UTR) 
of specific mRNAs and hence silence gene expression through either translational repression or direct mRNA degradation [5]. Such posttranscriptional gene regulation mechanisms allow miRNAs to control a wide range of biological processes, including cell proliferation and differentiation, migration, apoptosis, development and metabolism [6]. Accumulating evidence has shown aberrant miR-128 expression in tissues and blood from patients with many types of malignant tumors [7-12]. Volinia et al. examined 540 different types of malignant tumor samples by miRnome analysis and found significant increases in expression of miR-128 in colon, lung and pancreatic cancer [7]. In another report, in situ hybridization revealed that miR-128 expression is decreased in chemoresistant tumor tissues but increased in chemosensitive tissues, and the level of miR-128 expression in breast cancer tissues was correlated with patient response to novel adjuvant chemotherapy and survival [13].

Spectrin is a multifunctional protein. In addition to its primary role in maintaining the mechanical properties of cell membranes, it has been reported to be involved in many biological pathways such as the cell cycle, DNA repair, cell adhesion and spreading [14, 15]. Previous studies have demonstrated that nonerythroid $\alpha$ II spectrin ( $\alpha$ II Sp) is present in the mammalian cell nucleus, where it plays an important role in repair of DNA ICLs and is critical for chromosome stability [16-19]. $\alpha$ II Sp functions together with the DNA ICL repair proteins $\mathrm{XPF}$ and FANCA to localize at nuclear foci after DNA ICL damage $[16,18,20]$. A recent report has shown $\beta 2$ spectrin deficiency disturbs chromosome stability [21], and a number of studies have shown that spectrin is an essential regulator in a variety of cancers [22-26].

In the present study, we found that SPTAN1 is predicted to be a direct target of miR-128-3p. An inverse correlation between miR-128-3p expression and $\alpha \mathrm{II}$ Sp protein level in lung cancer treated with MMC was confirmed experimentally. MiR-128-3p was found to disrupt the cell cycle in lung cancer by targeting SPTAN1. Furthermore, the decreased level of $\alpha$ II Sp protein resulted in marked chromosomal instability and limited its function of recruiting XPF and FANCA for DNA repair.

\section{RESULTS}

\section{MMC-induced chromosomal aberrations and miR-128-3p expression in lung cancer cells}

In agreement with previous studies, MMC significantly induced chromosomal aberrations in lung cancer cells compared to a control group without MMC treatment (Figure 1A). To investigate the molecular mechanism by which MMC alters chromosomal stability to kill cancer cells, the levels of miR-128-3p and SPTAN1 expression were detected, as abundant data indicate their important functions in chromosomal instability, DNA ICLs and cancer $[12,16,27,28]$. As shown in Figure 1B, $1 \mathrm{C}$ and $1 \mathrm{E}$, the protein level of $\alpha \mathrm{II}$ Sp decreased by $47 \%$, whereas that of miR-128-3p increased by $\sim 1.4$-fold compared to the corresponding control. These results indicate that miR$128-3 p$ and $\alpha \mathrm{II} \mathrm{Sp}$ are reversely correlated. Importantly, mRNA expression of SPTAN1 was unchanged (Figure 1D).

\section{MiR-128-3p targets $S P T A N 1$ via translational repression}

MiRNAs are crucial regulators in lung cancer. Multiple target prediction programs were applied for determining the potential targets of miR-128-3p. Based on the species conservation and minimum free energy (MFE) of their binding sites as well as their cancer/DNA damage response correlations, SPTAN1 was highlighted for further investigation. Figure $1 \mathrm{~F}$ illustrates the predicted interaction of miR-128-3p and the target site in the SPTAN1 3'-UTR (MFE $=-27.9 \mathrm{kcal} / \mathrm{mol})$. A luciferase assay was performed to examine whether SPTAN1 is a direct target of miR-128-3p. The entire 3'-UTR of SPTAN1 placed in a reporter plasmid downstream of firefly luciferase. The resulting plasmid was transfected into A549 cells along with a transfection control plasmid and a miR-128-3p mimic or scrambled ncRNA. As hypothesized, compared to treatment with scrambled ncRNA, the miR-128-3p mimic decreased the luciferase activity to $35 \%$ of that of the reporter containing the miR-128-3p binding site, whereas a miR-128-3p inhibitor increased activity by $19 \%$. We generated mutations in the corresponding complementary seed sites in the $3^{\prime}$-UTR of SPTAN1 to eliminate the predicted miR-128-3p binding. Mutations in complementary seed sites almost fully rescued the repression of reporter activity caused by the miR-128-3p mimic (Figure 1G). Collectively, these findings strongly indicate that miR-128-3p can directly recognize the binding site in the 3 '-UTR of SPTAN1 and mediate posttranscriptional inhibition of the gene.

Theoretically, miRNAs silence gene expression by either translational repression or direct mRNA degradation. Thus, we next sought to confirm which mechanism miR-128-3p uses to modulate SPTAN1 expression. We transfected A549 cells with equal doses of scrambled ncRNA, miR-128-3p mimic or miR-128-3p inhibitor and analyzed SPTAN1 mRNA expression by RTPCR at $24 \mathrm{~h}$ post-transfection. SPTAN1 mRNA expression in all miR-128-3p mimic/inhibitor-transfected cells remained unchanged compared to that in all corresponding ncRNA-transfected cells (Figure 1H). However, we repeated the above experiments and determined whether 
overexpression or knockdown of miR-128-3p had an impact on the level of $\alpha$ II Sp protein by western blotting at $24 \mathrm{~h}$ post-transfection. Cells transfected with the miR128-3p mimic showed a level of $\alpha$ II Sp protein that was reduced to almost half of that of cells transfected with scrambled ncRNA; in contrast, the protein level of $\alpha$ II Sp increased by 30\% in miR-128-3p inhibitor-transfected cells compared to scrambled ncRNA-transfected cells (Figure 1I and 1J). We also transfected A549 cells with SPTAN1 siRNA and siRNA scramble, which showed a $51 \%$ decrease and the same effect as observed in the miR-128-3p mimic-transfected cells (Figure 1I and 1J). Similar results were obtained in H1975 lung cancer cells (Supplementary Figure S1). These findings demonstrate that miR-128-3p is capable of regulating SPTAN1 through translational repression.

\section{Up-regulation of miR-128-3p causes G2/M arrest and chromosomal instability}

To investigate whether miR-128-3p and SPTAN1 can affect the cell cycle after MMC-mediated DNA ICL damage, miR-128-3p was overexpressed or knocked down in A549 cells, and the cells were examined by flow cytometry (Figure 2A). Gain of function of miR-128-3p following mimic transfection resulted in an increase in the population of cells in $\mathrm{G} 2 / \mathrm{M}$ phase, with a concomitant decrease in the fraction of cells in $\mathrm{S}$ phase (Figure 2B). Figure 2A shows a representative experiment in which $32.87 \%$ of miR-128-3p scramble-treated cells were in G2/M phase but $38.98 \%$ of miR-128-3p mimic-treated cells were in $\mathrm{G} 2 / \mathrm{M}$ phase. Transfection with siRNA against $\alpha$ II Sp yielded an effect similar to that obtained

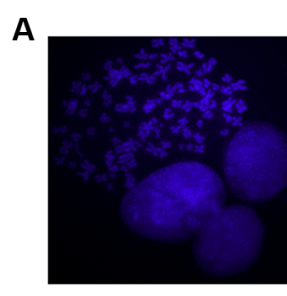

Control
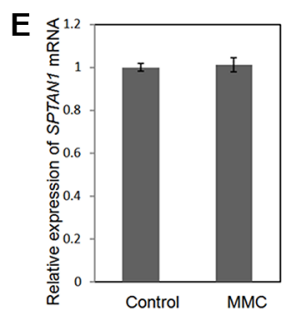

$\mathbf{F}$

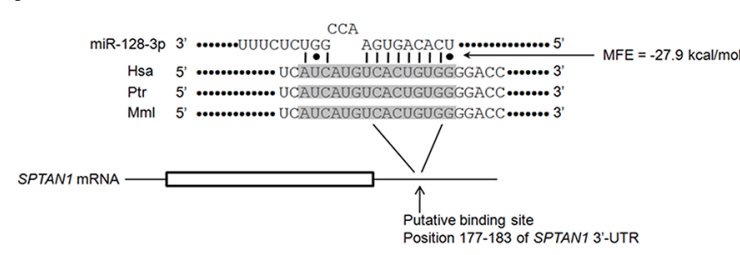

C

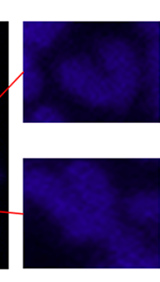

B

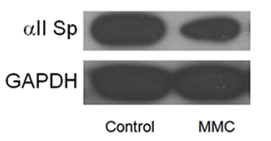

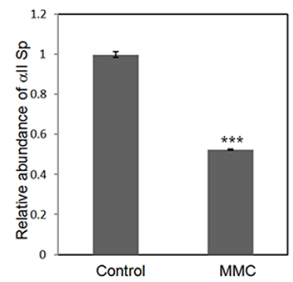

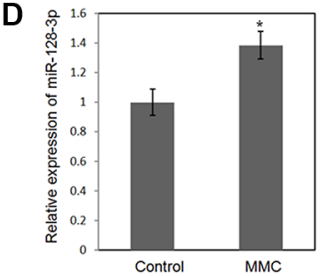

$\mathbf{G}$

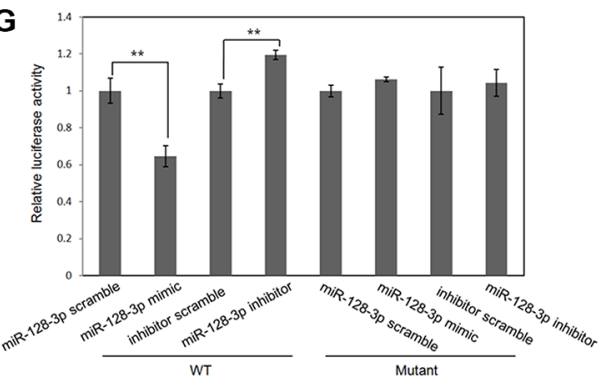

J

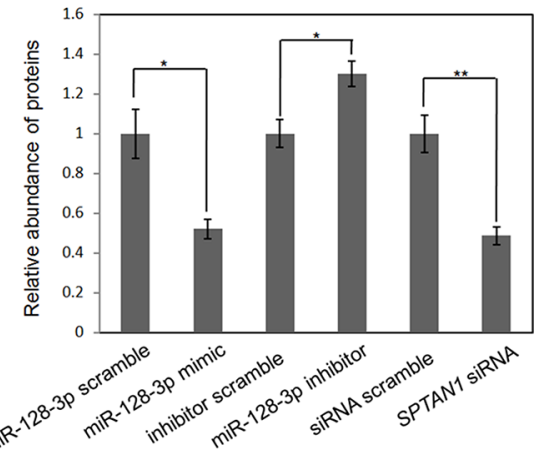

Figure 1: MiR-128-3p directly targets $\boldsymbol{S P T A N 1}$ via translational repression. (A) MMC treatment leads to an increase in chromosomal aberrations. (B, D and E) The effect of MMC on protein and mRNA levels of SPTAN1 and miR-128-3p expression. (C) Relative abundance of the $\alpha$ II Sp protein level in (B). (F) Schematic description of miR-128-3p binding site in the SPTAN1 3'-UTR. Paired bases are indicated by a black short line, and G:U pairs are indicated by dots. The predicted free energy of each hybrid is indicated. Sequence alignment of putative miR-128-3p binding sites across species are marked by a gray background. (G) A firefly luciferase reporter containing either wild-type (WT) or mutant SPTAN1 3'-UTR was co-transfected into A549 cells with scrambled ncRNA or miR-128-3p mimic or inhibitor. For comparison, luciferase activity in ncRNA-transfected cells was set at 1 . The y-axis shows arbitrary units representing the relative luciferase activity. $(\mathbf{H})$ Quantitative RT-PCR analysis of SPTAN1 mRNA levels in A549 cells transfected with scrambled ncRNA, miR-128-3p mimic or miR-128-3p inhibitor. (I) Western blot analysis of $\alpha$ II Sp protein levels in A549 cells transfected with scrambled ncRNAs, miR-128-3p mimic, miR-128-3p inhibitor or SPTAN1 siRNA. (J) Relative abundance of $\alpha$ II Sp in (I). The results are presented as the mean $\pm \mathrm{SE}$ of three independent experiments. ${ }^{*} p<0.05,{ }^{* *} p<0.01,{ }^{* * *} p<0.001$. 
via overexpression of miR-128-3p (Figure 2D). In the siRNA experiment, the G2/M-phase fractions of the siRNA scramble- and siRNA-treated groups were $32.49 \%$ and $43.83 \%$, respectively (Figure $2 \mathrm{~A}$ and 2D). In contrast, knockdown of miR-128-3p in A549 cells by a miR-128-3p inhibitor led to a reduction in cells in $\mathrm{G} 2 / \mathrm{M}$ phase (Figure 2C). The percentage of inhibitor scramble-treated cells in G2/M phase was $31.88 \%$, and that of miR-128-3p inhibitor-transfected cells was $27.6 \%$ (Figure 2A). The S-phase fractions of the control and miR-128-3p inhibitor groups were $27.8 \%$ and $38.69 \%$, respectively. These results indicate that miR-128-3p blocked cell cycle progression by arresting cells in $\mathrm{G} 2 / \mathrm{M}$ phase. Apoptosis analysis of lung cancer cells showed $2.8-8.1 \%$ apoptotic cells resulting from the same treatment.

To further identify the influence of miR-128-3p on chromosomal stability, overexpression or knockdown of miR-128-3p in A549 cells was evaluated for effects on chromosomal morphological changes using metaphase spread analysis. Metaphase spreads of A549 cells transfected with miR-128-3p mimic showed an obvious increase in chromosomal aberrations compared to ncRNAtransfected cells. SPTAN1 siRNA showed a similar effect as the miR-128-3p mimic. These results suggest that miR-128-3p promoted chromosome instability by silencing SPTAN1 (Figure 2E and 2F).

\section{MiR-128-3p destroys $\alpha$ II Sp/FANCA/XPF interaction by reducing $\alpha$ II Sp levels}

$\alpha$ II $\mathrm{Sp}$ is a crucial recruiter of FANCA and XPF in repair of DNA ICLs. To determine whether miR-128-3p influences interaction of the $\alpha$ II $\mathrm{Sp} / \mathrm{FANCA} / \mathrm{XPF}$ complex by inhibiting SPTAN1, immunofluorescent co-localization and co-immunoprecipitation (co-IP) experiments were performed. Figure 3A and 3B show an interruption of co-localization of FANCA or XPF with $\alpha$ II Sp in miR-128-3p mimic-transfected cells compared to ncRNA-transfected cells. Conversely, colocalization of FANCA or XPF with $\alpha$ II Sp in miR-128-3p inhibitor-transfected cells was greatly enhanced compared to that in inhibitor scramble-transfected cells. Knockdown of $\alpha$ II Sp by siRNA also led to loss of co-localization of FANCA or XPF with $\alpha$ II Sp (Figure 3A and 3B). Interestingly, FANCA protein levels in HeLa cells were reportedly stable in another study [17].

Co-IP were undertaken to ascertain whether miR-128-3p regulate the interaction between $\alpha$ II Sp and FANCA or XPF. As Figure 3C and 3D showed, cells transfected with miR-128-3p mimic made $58 \%, 27 \%$ or $46 \%$ reduction in protein levels of $\alpha$ II $\mathrm{Sp}, \mathrm{FANCA}$ or XPF respectively relative to the cells transfected with ncRNAs. Apparently, miR-128-3p inhibitor boosted the abundances of $\alpha$ II Sp, FANCA or XPF $(20 \%, 28 \%$ or $30 \%)$. SPTAN1 siRNA and miR-128-3p mimic have similar effects to their interaction of the complex. Thus, these results indicate miR-128-3p breaks the formation of $\alpha$ II Sp/FANCA/XPF complex by eliminating $\alpha$ II Sp.

\section{DISCUSSION}

MMC exhibits activity against NSCLC, a mechanism that involves cross-linking of inter-strand DNA to prevent DNA replication and inhibit tumor cell division. As a structural protein, $\alpha \mathrm{II} \mathrm{Sp}$ is implicated in the repair of DNA ICLs. However, the relationship between miRNAs and SPTAN1 in DNA repair and the potential function by which MMC inhibits cancer cells is poorly understood. In this study, we describe a novel function for miR-128-3p, whereby regulation of chromosomal stability and cell cycle progression occur through SPTAN1 in lung cancer cells treated with MMC. This conclusion is supported by several lines evidence: increased expression of miR-128-3p and decreased expression of SPTAN1 in lung cancer cells treated with MMC; a putative SPTAN1 binding site in the $3^{\prime}$-UTR that is subject to miR-128-3p regulation; overexpression of miR-128-3p augmented MMC-mediated chromosomal instability and cell cycle arrest of G2/M phase in lung cancer cells; these biological dysfunctions were caused by disruption of the $\alpha$ II Sp/FANCA/XPF complex induced by miR-128-3p. Completely different from the traditional function of DNA ICLs caused by MMC, the miR-128-3p-SPTAN1 axis is a novel molecular mechanism for inhibiting the repair of DNA ICLs and could serve as an excellent potential auxiliary to treat lung cancer.

It has been found that aberrant expression of miR-128 occurs in malignant tumors, and this miRNA has been identified as a key regulator of oncogenic properties. Reduced miR-128 was first found in glioblastoma [29], and miR-128 is involved in multiple signal pathways associated with head and neck squamous cell carcinoma progression and growth [30]. MiR-128 is also down-regulated in glioma tissue and serum and could be used as potential biomarker in glioma identification, early diagnosis, classification and prognosis prediction [31]. MiR-128 targets ZEB1 in prostate cancer, and the miR-128-ZEB1 axis could be a promising prognostic and therapeutic target for future prostate cancer therapy. MiR-128 promotes proliferation in osteosarcoma cells by downregulating PTEN [32]. Abnormal expression of miR-128 contributes to the malignant phenotypes of cancer cells, such as proliferation [33], cell motility, invasion [34, 35], apoptosis [36] and self-renewal [37]. Here, we note that overexpression of miR-128 remarkably hindered DNA repair to contribute to the anti-cancer function of MMC and sensitized MMC-mediated chemotherapy in lung cancer.

It is well believed that MMC is an important effector of cell cycle arrest and chromosomal stability. For example, the percentage of cells containing chromosomal aberrations was significantly higher among PHF9-knock-down cells than among control cells in the presence of MMC [38]. Previous studies have shown that $\mathrm{MMC}$ causes a significant increase in the number 


\section{A}
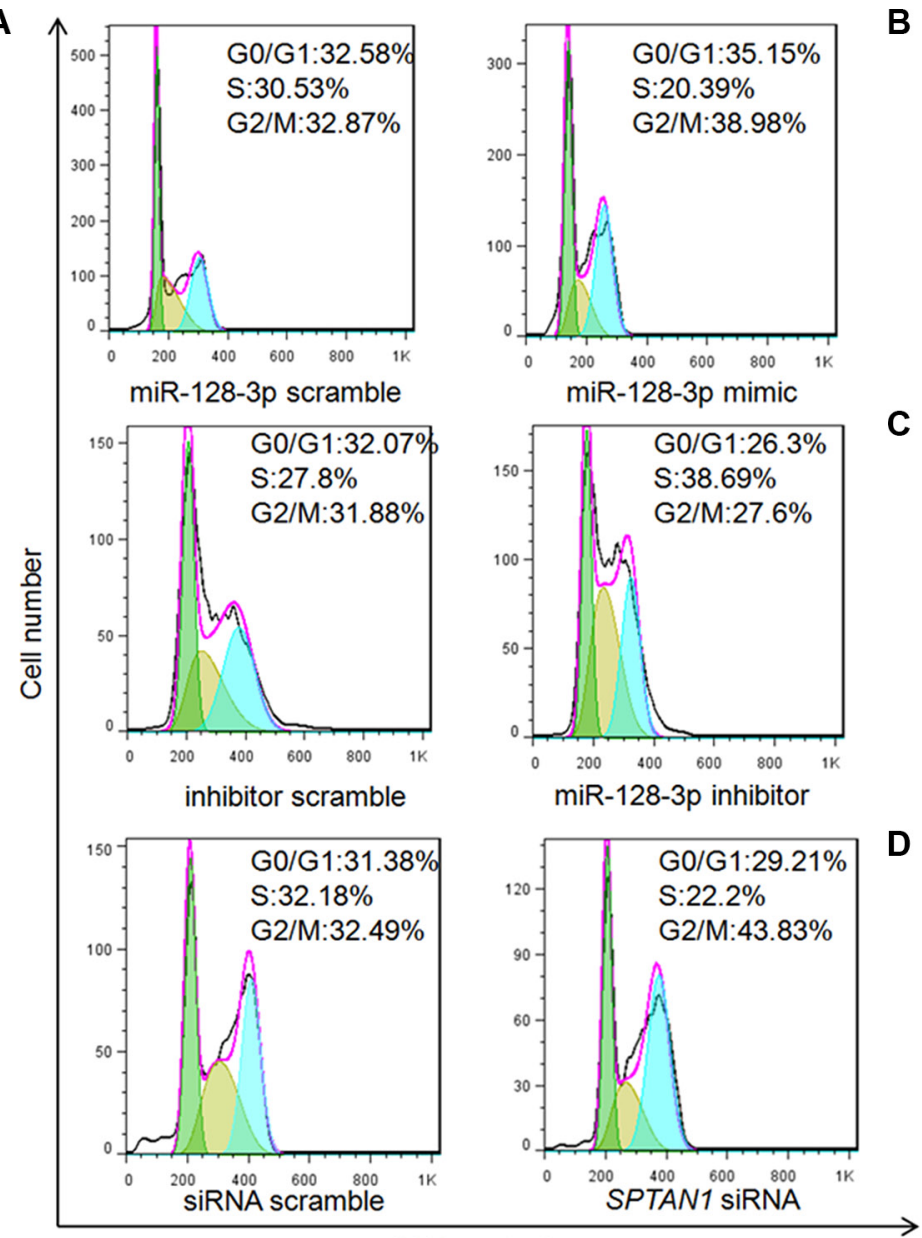

E

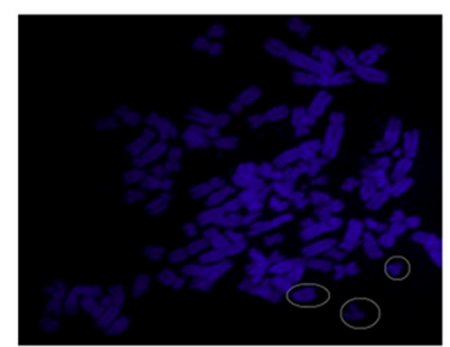

miR-128-3p scramble

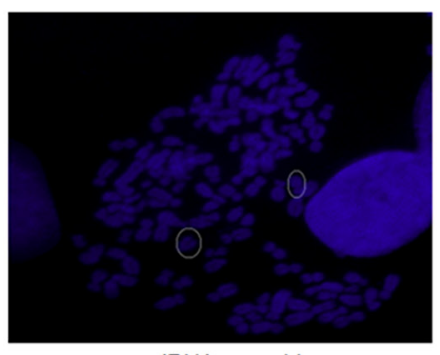

siRNA scramble

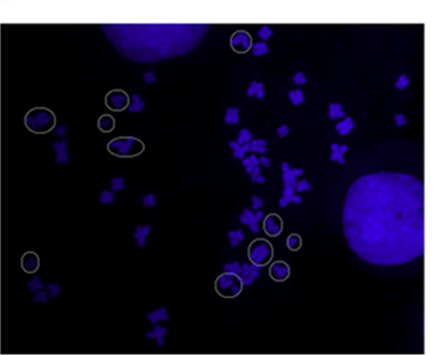

miR-128-3p mimic

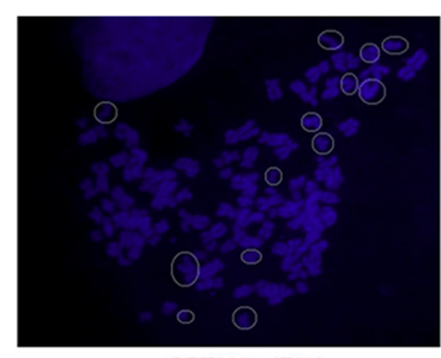

SPTAN1 SIRNA

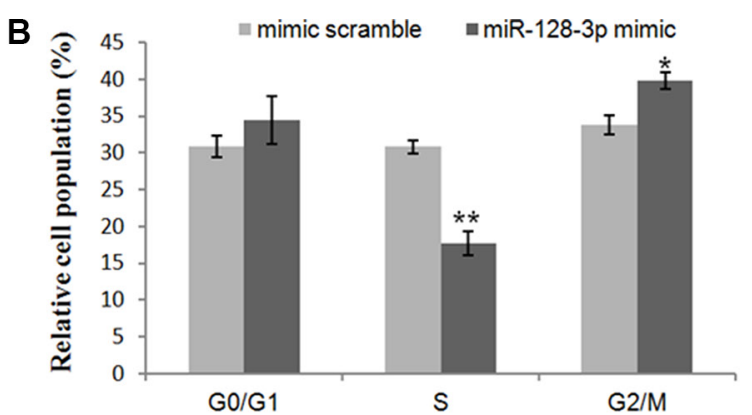

C
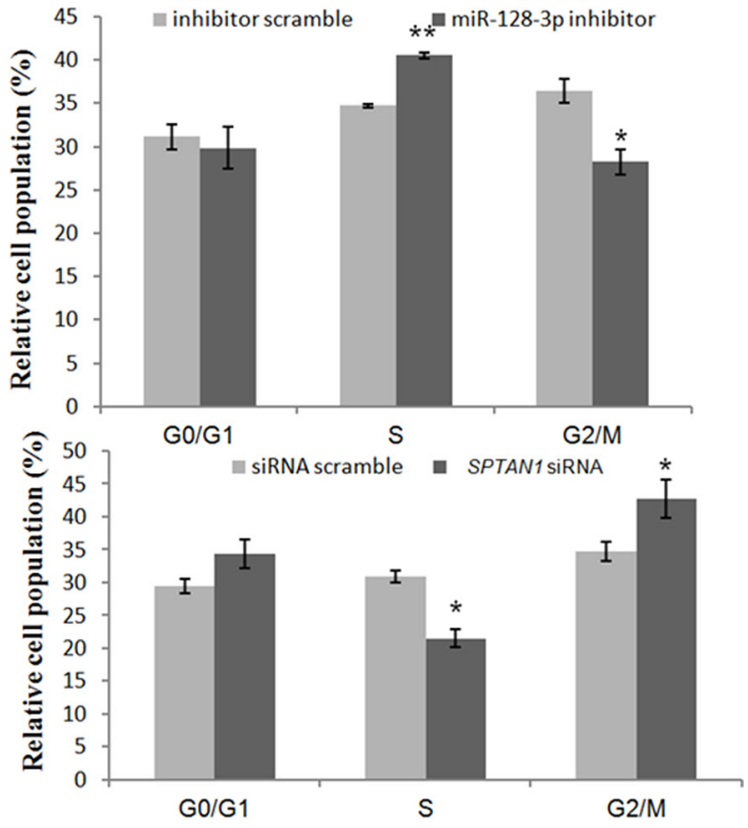

$\mathbf{F}$

Figure 2: MiR-128-3p regulates the cell cycle and chromosomal aberrations. (A) A549 cells were transfected with synthetic RNAs and subsequently treated with MMC; the percentages of cells in G0/G1, S and G2/M phases were determined by flow cytometry. (B, C and D) Bar graphs illustrate quantification of the cell cycle. (E) The effect of scrambled ncRNAs, miR-128-3p mimic and SPTANI siRNA on chromosomes in A549 cells. Circles indicate chromosomal aberrations. (F) One hundred metaphase spreads were scored for chromosomal aberrations, and the average number of chromosomal aberrations per metaphase from three independent experiments is shown. The results are presented as the mean $\pm \mathrm{SE}$ of three independent experiments. ${ }^{*} p<0.05,{ }^{* *} p<0.01$. 
of chromosomal aberrations, which could be related to a defect in the repair of DNA ICLs [27]. It has been reported that Bid-deficient cells have a defect in S or G2/M phase cell-cycle arrest upon treatment with replicative stressinducing drugs such as MMC [39]. MMC also induces cell cycle arrest at clinically relevant doses $(1 \mu \mathrm{g} / \mathrm{ml})$ [40]. In addition to the above-mentioned findings, we here present the first evidence that miR-128-3p enhances $\mathrm{MMC}$-induced chromosomal aberrations and cell cycle arrest at G2/M phase by targeting SPTAN1. Both miR128-3p and $\alpha$ II Sp are essential for the function of MMC in causing chromosomal aberrations and cell cycle arrest.

Fanconi Anaemia (FA) is an inherited recessive disease caused by mutations in at least 15 different genes. Cellular hypersensitivity to cross-linking agents such as MMC is observed in FA, a trait that has been used as a diagnostic marker for FA patients. There is also biochemical evidence showing that FANCA, FANCC and FANCG bind in vitro to psoralen cross-linked DNA in a complex with human $\alpha$ II Sp [18]. Moreover, $\alpha$ II Sp is present in the mammalian cell nucleus, where it has an important function in DNA ICL repair and chromosome stability. $\alpha$ II Sp has been shown to recruit DNA repair-associated proteins (FANCA and XPF) to nuclear foci induced by DNA ICLs and to be crucial for chromosome stability [18, 20, 27]. Formation of the $\alpha \mathrm{II} \mathrm{Sp} / \mathrm{FANCA} / \mathrm{XPF}$ complex is involved in initial damage recognition and the incision steps of the repair process $[20,41,42]$. To date, however, there is no evidence that miRNAs participate in $\alpha$ II $\mathrm{Sp}$ regulation or adjust its function in lung cancer chemotherapy. Here, we demonstrate that overexpression of miR-128-3p downregulates SPTAN1 expression via translational repression and results in the failure to recruit FANCA and XPF to facilitate chromosomal instability after MMC-induced DNA ICL damage in lung cancer cells. MMC treatment not only cross-links DNA but also inhibits expression of
A

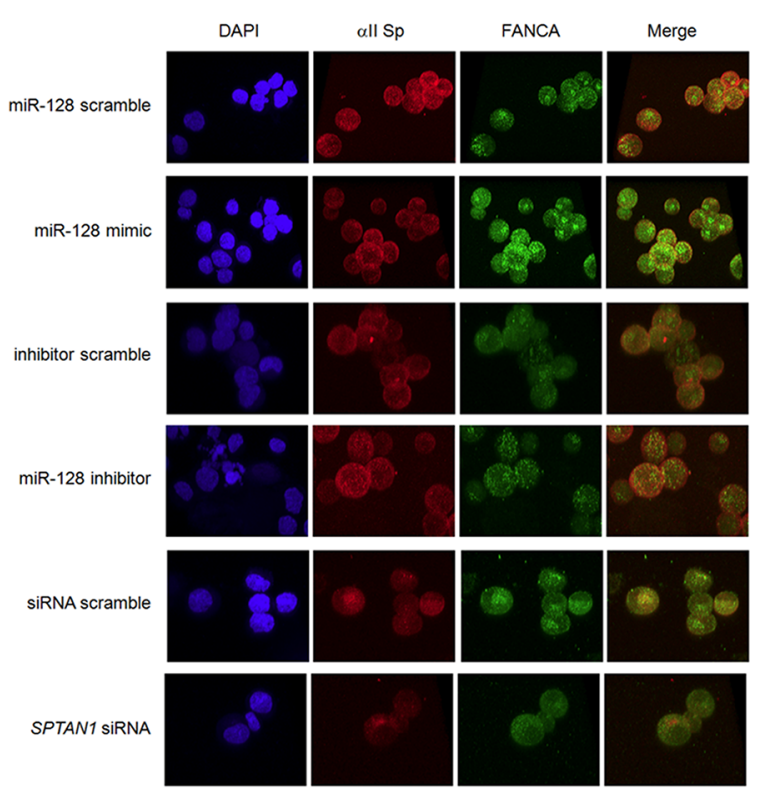

C

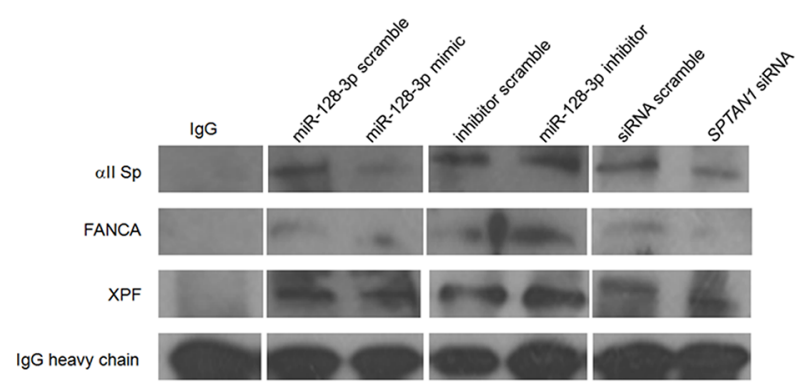

B

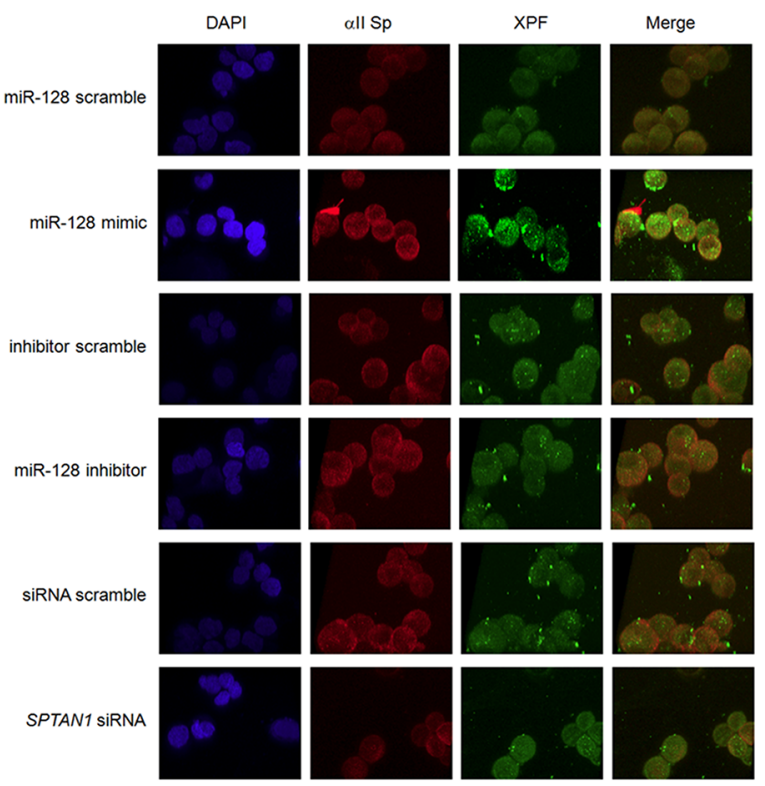

D

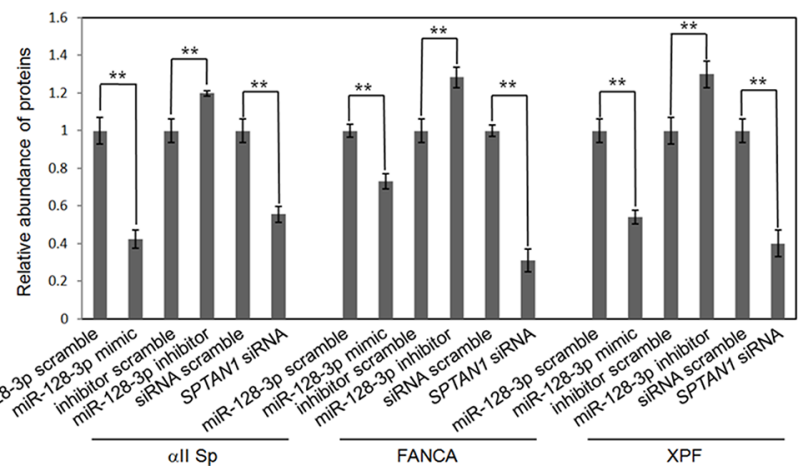

Figure 3: MiR-128-3p disrupts co-localization and interaction of FANCA, XPF and $\alpha$ II Sp. (A) Co-localization of FANCA and $\alpha$ II Sp. Cells were stained with anti- $\alpha$ II Sp (red) and anti-FANCA (green) antibodies, and nuclear DNA was counterstained with DAPI (blue). (B) Co-localization of XPF and $\alpha$ II Sp. Cells were stained with anti- $\alpha$ II Sp (red) and anti-XPF (green) antibodies, and nuclear DNA was counterstained with DAPI (blue). (C) Interaction of $\alpha$ II Sp, XPF and FANCA. Co-IP was carried out using an anti- $\alpha$ II Sp antibody or IgG (as a control), and western blot analyses were performed using an anti- $\alpha$ II Sp, anti-FANCA or XPF antibody. (D) Bar graphs show the relative abundances of $\alpha$ II Sp, FANCA or XPF. The results are presented as the mean \pm SE of three independent experiments. ${ }^{* *} p<0.01$. 
SPTAN1 through miR-128-3p to disrupt recruitment of DNA repair-associated proteins (FANCA and XPF), with dual destructive effects in lung cancer cells. This is a novel pathway for interrupting repair of DNA ICLs to enhance the anti-cancer function of MMC.

Although FA proteins can stabilize $\alpha$ II $\mathrm{Sp}$, in FA cells, which have significant $\alpha$ II Sp deficiency, $\alpha$ II Sp can be cleaved into small segments by $\mu$-calpain, but $\alpha$ II $\mathrm{Sp}$ is not affected by alternative RNA splicing $[19,43]$. However, in both normal and cancer cells, changes in the level of $\alpha$ II Sp protein induced by MMC should be caused by a novel miRNA-related pathway different from the above-mentioned $\mu$-calpain degradation system. Additionally, the anti- $\alpha$ II Sp antibody used in this study detected $\alpha \mathrm{II} \mathrm{Sp}$ in the cytoplasm and nucleus; thus, in-depth analysis is required in the near future. In addition, it remains unclear whether the these SPTAN1 regulation pathways cooperate together to function under certain conditions. MiRNAs have potential pleiotropic effects, and many miRNAs can influence a single target gene. Therefore, other miRNAs or other miR-128-3p target genes might be involved in this process.

Taken together, we extend the current knowledge by highlighting the role of miR-128-3p in the sensitivity of lung cancer to chemotherapy. The miR-128-3p-SPTAN1 axis provides a novel avenue for understanding the mechanism of chemosensitivity, and miR-128-3p could be a candidate molecular target for improving the efficacy of lung cancer chemotherapy.

\section{MATERIALS AND METHODS}

\section{Cell culture and treatment of cells with MMC}

A549 human lung adenocarcinoma cell line was purchased from the Shanghai Institute of Cell Biology, Chinese Academy of Sciences (Shanghai, China). The A549 cells were cultured in DMEM supplemented with $10 \%$ fetal bovine serum (FBS) (GIBCO). All cells were incubated in a $5 \% \mathrm{CO}_{2}, 37^{\circ} \mathrm{C}$, water-saturated atmosphere. Synthetic RNAs-transfected cells were treated with $400 \mathrm{nM}$ MMC (Sigma-Aldrich, USA) $24 \mathrm{~h}$ after transfection, as previously described [27].

\section{Overexpression or knockdown of miR-128-3p}

Synthetic miR-128-3p mimic, miR-128-3p inhibitor and scrambled negative control RNAs (mimic scramble and inhibitor scramble) were purchased from GenePharma (Shanghai, China). Cells were seeded in 6-well plates and were transfected with the following day using Lipofectamine 2000 (Invitrogen, Carlsbad, CA, USA), according to the manufacturer's instructions. For each well, equal doses of miR-128-3p mimic, miR-128-3p inhibitor or scrambled ncRNA were added. Cells were collected $24 \mathrm{~h}$ after transfection for further analysis.

\section{RNA isolation and quantitative RT-PCR}

Total RNA was extracted from the cultured cells using TRIzol Reagent (Invitrogen, Carlsbad, CA, USA) according to the manufacturer's instructions. For quantitative RTPCR analysis of SPTAN1 and GAPDH, $1 \mu \mathrm{g}$ of total RNA was reverse transcribed to cDNA with oligo (dT) and Thermoscript (TaKaRa). Real-time PCR for SPANTI and GAPDH was performed on an Applied Biosystems 7300 Sequence Detection System (Applied Biosystems) using SYBR green dye (Invitrogen, Carlsbad, CA, USA). The sequences of the sense and antisense primers used for amplification of SPTAN1 and GAPDH were as follows: SPTAN1 (sense): 5'-TACGAGAATGTGAGGACGTGA-3', SPTAN1 (antisense): 5'-CATGAGCAGCCATATCTGTTTGA-3'; GAPDH (sense): 5'-GATATTGTTGCCATCAATGAC-3', GAPDH (antisense): 5'-TTGATTTTGGAGGGATCT CG-3'.

Assays to quantify mature miR-128-3p were carried out using TaqMan miRNA probes (Applied Biosystems). Briefly, 1 $\mu \mathrm{g}$ of total RNA was reverse-transcribed to cDNA using AMV reverse transcriptase (TaKaRa) and a stem-loop RT primer (Applied Biosystems). Real-time PCR was performed using a TaqMan PCR kit on an Applied Biosystems 7300 Sequence Detection System (Applied Biosystems). All reactions, including no-template controls, were run in triplicate. After the reactions, the $\mathrm{CT}$ values were determined using fixed threshold settings. In the experiments presented here, miRNA expression in cells was normalized to U6. The relative amount of miR128-3p to internal control U6 was calculated with the equation $2^{-\Delta \mathrm{C}}$, in which $\Delta \mathrm{C}_{\mathrm{T}}=\mathrm{C}_{\mathrm{T} \text { miRNA }}-\mathrm{C}_{\mathrm{T} \text { U6 }}$.

\section{Target predictions}

Target genes were predicted with three algorithms from TargetScan (http://genes.mit.edu/targetscan/), miRWalk (http://www.umm.uni-heidelberg.de/apps/zmf/mirwalk/ index.html) and miRanda (http://www.microrna.org).

\section{Plasmid construction and luciferase assay}

The entire 3'-UTR or mutant of SPANTI (Invitrogen, Carlsbad, CA, USA) were inserted into the p-MIR-report plasmid (Ambion). For luciferase reporter assays, A549 cells were cultured in 24-well plates, and each well was transfected with $1 \mu \mathrm{g}$ of firefly luciferase reporter plasmid, $1 \mu \mathrm{g}$ of $\beta$-galactosidase expression vector (Ambion), and equal amounts of scrambled ncRNA, miR-128-3p mimic, or miR-128-3p inhibitor using Lipofectamine 2000 (Invitrogen, Carlsbad, CA, USA). The $\beta$-galactosidase vector was used as a transfection control. At $24 \mathrm{~h}$ post-transfection, cells were assayed using luciferase assay kits (Promega). Data depicted are representative of three independent experiments.

\section{Protein extraction and western blotting}

Whole cell extracts was obtained at $24 \mathrm{~h}$ posttransfection. All protein level was quantified by western 


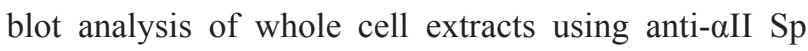
antibody (Santa Cruz Biotechnology). These samples were normalized by probing the same blots with a GAPDH antibody.

\section{Cell cycle analysis}

Cells were harvested and washed twice in PBS at room temperature and resuspended at $2 \times 10^{6} \mathrm{cells} / \mathrm{ml}$ in PBS. For propidium iodide staining, washed cells were fixed in $70 \%$ ethanol at $-20^{\circ} \mathrm{C}$ overnight and the rest of the steps were performed according to the manufacture's procedure (Molecular Probes Inc.). Cells were washed twice in PBS, resuspended in FACS buffer (PBS, 0.2\% BSA and $1 \%$ sodium azide) and analyzed using a FACS flow cytometer (BD Biosciences, San Jose, CA).

\section{Apoptosis}

Cell apoptosis was determined by an BD Biosciences Annexin V Apoptosis detection kit. Briefly, cells were collected and resuspended in $0.5 \mathrm{~mL}$ of binding buffer and incubated with annexin V-FITC and propidium iodide for $10 \mathrm{~min}$ in the dark at room temperature and analyzed using a FACS flow cytometer (BD Biosciences, San Jose, CA).

\section{Co-IP of proteins and immunoblot analysis}

Cells were lysed with lysis buffer $(20 \mathrm{mM}$ Tris$\mathrm{HCl}, 150 \mathrm{mM} \mathrm{NaCl}, 0.5 \%$ Nonidet P-40, $2 \mathrm{mM}$ EDTA, $0.5 \mathrm{mM}$ DTT, $1 \mathrm{mM} \mathrm{NaF}, 1 \mathrm{mM}$ PMSF and $1 \%$ Protease Inhibitor Cocktail from Sigma, $\mathrm{pH} 7.5$ ) for $30 \mathrm{~min}$ on ice. The lysates were cleared by centrifugation $(16,000 \mathrm{~g})$ for $10 \mathrm{~min}$ at $4^{\circ} \mathrm{C}$ and then immunoprecipitated with anti- $\alpha$ II Sp antibody or IgG followed by protein G-Agarose beads. After the elution from the beads, levels of aII Sp, FANCA and XPF were analyzed by western blot.

\section{Metaphase spreads and chromosome analysis}

Cells transfected with scrambled ncRNA, miR-128$3 \mathrm{p}$ mimic, miR-128-3p inhibitor or SPTAN1 siRNA were incubated for $24 \mathrm{~h}$, and subsequently treated with MMC and incubated for an additional $24 \mathrm{~h}$. Colcemid $(0.1 \mu \mathrm{g} / \mathrm{ml})$ (Sigma-Aldrich, USA) was added $22 \mathrm{~h}$ after MMC treatment and incubation continued for $2 \mathrm{~h}$. The cells were harvested, swollen in hypotonic solution, fixed and slides stained with 4'-6' diamidino-2-phenylindole (DAPI). At least 100 metaphases from each group were scored for chromosomal aberrations. All fluorescence images were captured on a Nikon confocal microscope (Nikon, Tokyo, Japan) using a $\times 100$ oil immersion lens and analyzed using Nis-element advanced research software (Nikon, Tokyo, Japan).

\section{Immunofluorescence}

A549 cells were transfected with scrambled ncRNA, miR-128-3p mimic, miR-128-3p inhibitor or SPTAN1 siRNA, and subsequently treated ( $24 \mathrm{~h}$ post transfection) with MMC. Then cells were applied to poly- $L$-lysine coated chamber slides and allowed to attach for 40 minutes at $37^{\circ} \mathrm{C}$. They were then fixed with $4 \%$ paraformaldehyde for 20 minutes, washed with PBS and permeabilized with $0.2 \%$ Triton-X100 in PBS for 10 minutes at room temperature. The cells were then blocked in 10\% FBS for 1 hours. The primary antibody of $\alpha$ II Sp, XPF or FANCA (Santa Cruz Biotechnology) was then added and allowed to bind for 12 hours at $4^{\circ} \mathrm{C}$. After 5-minute washes with PBS, the appropriate secondary antibody was added: Alexafluor 488 mouse anti-mouse $\mathrm{IgG}$ conjugate (Invitrogen, Carlsbad, CA, USA), Alexafluor 594 goat anti-rabbit IgG conjugate (Invitrogen, Carlsbad, CA, USA) or Alexafluor 594 donkey anti-goat IgG conjugate (Invitrogen, Carlsbad, CA, USA). Incubation with the secondary antibodies was carried out for $1 \mathrm{~h}$ at room temperature in the dark and then washed with PBS for three times. The slides were then mounted with cover slips using an aqueous anti-fade mounting agent (Molecular Probes Inc.). For those cells which were examined with a DNA counter stain, after the last antibody labelling step, the cells were treated with DAPI. All fluorescence images were captured on a Nikon confocal microscope (Nikon, Tokyo, Japan) as z-stacks using a $\times 100$ oil immersion lens and analyzed using Nis-element advanced research software (Nikon, Tokyo, Japan). Stacks were separated by $0.2-0.5 \mathrm{~mm}$. All immunofluorescence staining experiments were repeated in triplicate, including negative controls to determine the background staining.

\section{Statistical analysis}

All experiments were repeated three to five times. Data shown are presented as mean \pm SE. Statistical significance was considered at $p<0.05$ using the Student's $t$-test.

\section{ACKNOWLEDGMENTS}

We are grateful to Hao Yang for kindly initiating the work.

\section{CONFLICTS OF INTEREST}

The authors declare no conflicts of interest.

\section{GRANT SUPPORT}

This work was supported by the National Basic Research Program of China (973 Program, 
2014CB542300, 2012CB517603 and 2011CB504803); the National Science Fund for Distinguished Young Scholars (81025019); the National Natural Science Foundation of China (31271378, 81101330, 81250044, 31470716, 91213306, 31000323 and 31070672); the Natural Science Foundation of Jiangsu Province (BK2011013 and BK20131272); the Specialized Research Fund for the Doctoral Program of Higher Education of China (20100091120023); and the Fundamental Research Funds for the Central Universities (1095020823).

\section{REFERENCES}

1. Sculier JP, Ghisdal L, Berghmans T, Branle F, Lafitte JJ, Vallot F, Meert AP, Lemaitre F, Steels E, Burniat A, Mascaux C, European Lung Cancer Working P. The role of mitomycin in the treatment of non-small cell lung cancer: a systematic review with meta-analysis of the literature. Br J Cancer. 2001; 84:1150-1155.

2. Bakowski MT, Crouch JC. Chemotherapy of non-small cell lung cancer: a reappraisal and a look to the future. Cancer Treat Rev. 1983; 10:159-172.

3. Tomasz M, Palom Y. The mitomycin bioreductive antitumor agents: cross-linking and alkylation of DNA as the molecular basis of their activity. Pharmacol Ther. 1997; 76:73-87.

4. Palom Y, Suresh Kumar G, Tang LQ, Paz MM, Musser SM, Rockwell S, Tomasz M. Relative toxicities of DNA crosslinks and monoadducts: new insights from studies of decarbamoyl mitomycin $\mathrm{C}$ and mitomycin C. Chem Res Toxicol. 2002; 15:1398-1406.

5. Pasquinelli AE. MicroRNAs and their targets: recognition, regulation and an emerging reciprocal relationship. Nat Rev Genet. 2012; 13:271-282.

6. Pelaez N, Carthew RW. Biological robustness and the role of microRNAs: a network perspective. Curr Top Dev Biol. 2012; 99:237-255.

7. Volinia S, Calin GA, Liu CG, Ambs S, Cimmino A, Petrocca F, Visone R, Iorio M, Roldo C, Ferracin M, Prueitt RL, Yanaihara N, Lanza G, et al. A microRNA expression signature of human solid tumors defines cancer gene targets. Proc Natl Acad Sci USA. 2006; 103:2257-2261.

8. Roth P, Wischhusen J, Happold C, Chandran PA, Hofer S, Eisele G, Weller M, Keller A. A specific miRNA signature in the peripheral blood of glioblastoma patients. J Neurochem. 2011; 118:449-457.

9. Novakova J, Slaby O, Vyzula R, Michalek J. MicroRNA involvement in glioblastoma pathogenesis. Biochem Biophys Res Commun. 2009; 386:1-5.

10. Pang JC, Kwok WK, Chen Z, Ng HK. Oncogenic role of microRNAs in brain tumors. Acta Neuropathol. 2009; 117:599-611.

11. Lages E, Guttin A, El Atifi M, Ramus C, Ipas H, Dupre I, Rolland D, Salon C, Godfraind C, deFraipont F, Dhobb M,
Pelletier L, Wion D, et al. MicroRNA and target protein patterns reveal physiopathological features of glioma subtypes. PLoS One. 2011; 6:e20600.

12. Venkataraman S, Alimova I, Fan R, Harris P, Foreman N, Vibhakar R. MicroRNA 128a increases intracellular ROS level by targeting Bmi-1 and inhibits medulloblastoma cancer cell growth by promoting senescence. PLoS One. 2010; 5:e10748.

13. Zhu Y, Yu F, Jiao Y, Feng J, Tang W, Yao H, Gong C, Chen J, Su F, Zhang Y, Song E. Reduced miR-128 in breast tumorinitiating cells induces chemotherapeutic resistance via Bmi-1 and ABCC5. Clin Cancer Res. 2011; 17:7105-7115.

14. Machnicka B, Grochowalska R, Boguslawska DM, Sikorski AF, Lecomte MC. Spectrin-based skeleton as an actor in cell signaling. Cell Mol Life Sci. 2012; 69:191-201.

15. Zhang R, Zhang C, Zhao Q, Li D. Spectrin: structure, function and disease. Sci China Life Sci. 2013; 56:1076-1085.

16. Sridharan D, Brown M, Lambert WC, McMahon LW, Lambert MW. Nonerythroid $\alpha$ II spectrin is required for recruitment of FANCA and XPF to nuclear foci induced by DNA interstrand cross-links. J Cell Sci. 2003; 116:823-835.

17. McMahon LW, Zhang P, Sridharan DM, Lefferts JA, Lambert MW. Knockdown of $\alpha$ II spectrin in normal human cells by siRNA leads to chromosomal instability and decreased DNA interstrand cross-link repair. Biochem Biophys Res Commun. 2009; 381:288-293.

18. McMahon LW, Sangerman J, Goodman SR, Kumaresan K, Lambert MW. Human $\alpha$ spectrin II and the FANCA, FANCC, and FANCG proteins bind to DNA containing psoralen interstrand cross-links. Biochemistry. 2001; 40:7025-7034.

19. Lambert MW. Functional Significance of Nuclear $\alpha$ Spectrin. J Cell Biochem. 2015; 1816-1830.

20. McMahon LW, Walsh CE, Lambert MW. Human $\alpha$ spectrin II and the Fanconi anemia proteins FANCA and FANCC interact to form a nuclear complex. J Biol Chem. 1999; 274:32904-32908.

21. Horikoshi N, Pandita RK, Mujoo K, Hambarde S, Sharma D, Mattoo AR, Chakraborty S, Charaka V, Hunt CR, Pandita TK. $\beta 2$-spectrin depletion impairs DNA damage repair. Oncotarget. 2016; 7:33557-33570.

22. Macoska JA, Xu J, Ziemnicka D, Schwab TS, Rubin MA, Kotula L. Loss of expression of human spectrin src homology domain binding protein 1 is associated with $10 \mathrm{p}$ loss in human prostatic adenocarcinoma. Neoplasia. 2001; 3:99-104.

23. Yao ZX, Jogunoori W, Choufani S, Rashid A, Blake T, Yao W, Kreishman P, Amin R, Sidawy AA, Evans SR, Finegold M, Reddy EP, Mishra B, et al. Epigenetic silencing of $\beta$-spectrin, a TGF- $\beta$ signaling/scaffolding protein in a human cancer stem cell disorder: Beckwith-Wiedemann syndrome. J Biol Chem. 2010; 285:36112-36120.

24. Baek HJ, Pishvaian MJ, Tang Y, Kim TH, Yang S, Zouhairi ME, Mendelson J, Shetty K, Kallakury B, 
Berry DL, Shin KH, Mishra B, Reddy EP, et al. Transforming growth factor- $\beta$ adaptor, $\beta 2$-spectrin, modulates cyclin dependent kinase 4 to reduce development of hepatocellular cancer. Hepatology. 2011; 53:1676-1684.

25. Kim SS, Shetty K, Katuri V, Kitisin K, Baek HJ, Tang Y, Marshall B, Johnson L, Mishra B, Mishra L. TGF-beta signaling pathway inactivation and cell cycle deregulation in the development of gastric cancer: role of the $\beta$-spectrin, ELF. Biochem Biophys Res Commun. 2006; 344:1216-1223.

26. Kitisin K, Ganesan N, Tang Y, Jogunoori W, Volpe EA, Kim SS, Katuri V, Kallakury B, Pishvaian M, Albanese C, Mendelson J, Zasloff M, Rashid A, et al. Disruption of transforming growth factor-beta signaling through $\beta$-spectrin ELF leads to hepatocellular cancer through cyclin D1 activation. Oncogene. 2007; 26:7103-7110.

27. Zhang P, Herbig U, Coffman F, Lambert MW. Nonerythroid $\alpha$ spectrin prevents telomere dysfunction after DNA interstrand cross-link damage. Nucleic Acids Res. 2013; 41:5321-5340.

28. Li ML, Fu WM, Wo LL, Shu XH, Liu F, Li CG. miR-128 and its target genes in tumorigenesis and metastasis. Exp Cell Res. 2013; 319:3059-3064.

29. Ciafre SA, Galardi S, Mangiola A, Ferracin M, Liu CG, Sabatino G, Negrini M, Maira G, Croce CM, Farace MG. Extensive modulation of a set of microRNAs in primary glioblastoma. Biochem Biophys Res Commun. 2005; 334:1351-1358.

30. Hauser B, Zhao Y, Pang X, Ling Z, Myers E, Wang P, Califano $\mathrm{J}, \mathrm{Gu} \mathrm{X}$. Functions of miRNA-128 on the regulation of head and neck squamous cell carcinoma growth and apoptosis. PLoS One. 2015; 10:e0116321.

31. Sun J, Liao KM, Wu XC, Huang J, Zhang S, Lu XJ. Serum microRNA-128 as a biomarker for diagnosis of glioma. Int J Clin Exp Med. 2015; 8:456-463.

32. Shen L, Chen XD, Zhang YH. MicroRNA-128 promotes proliferation in osteosarcoma cells by downregulating PTEN. Tumour Biol. 2014; 35:2069-2074.

33. Zhang Y, Chao T, Li R, Liu W, Chen Y, Yan X, Gong Y, Yin B, Liu W, Qiang B, Zhao J, Yuan J, Peng X. MicroRNA-128 inhibits glioma cells proliferation by targeting transcription factor E2F3a. J Mol Med. 2009; 87:43-51.

34. Evangelisti C, Florian MC, Massimi I, Dominici C, Giannini G, Galardi S, Bue MC, Massalini S, McDowell HP,
Messi E, Gulino A, Farace MG, Ciafre SA. MiR-128 upregulation inhibits Reelin and DCX expression and reduces neuroblastoma cell motility and invasiveness. FASEB J. 2009; 23:4276-4287.

35. Khan AP, Poisson LM, Bhat VB, Fermin D, Zhao R, Kalyana-Sundaram S, Michailidis G, Nesvizhskii AI, Omenn GS, Chinnaiyan AM, Sreekumar A. Quantitative proteomic profiling of prostate cancer reveals a role for miR-128 in prostate cancer. Mol Cell Proteomics. 2010; 9:298-312.

36. Adlakha YK, Saini N. MicroRNA-128 downregulates Bax and induces apoptosis in human embryonic kidney cells. Cell Mol Life Sci. 2011; 68:1415-1428.

37. Godlewski J, Nowicki MO, Bronisz A, Williams S, Otsuki A, Nuovo G, Raychaudhury A, Newton HB, Chiocca EA, Lawler S. Targeting of the Bmi-1 oncogene/stem cell renewal factor by microRNA-128 inhibits glioma proliferation and self-renewal. Cancer Res. 2008; 68:9125-9130.

38. Meetei AR, de Winter JP, Medhurst AL, Wallisch M, Waisfisz Q, van de Vrugt HJ, Oostra AB, Yan ZJ, Ling C, Bishop CE, Hoatlin ME, Joenje H, Wang WD. A novel ubiquitin ligase is deficient in Fanconi anemia. Nat Genet. 2003; 35:165-170.

39. Kaufmann T, Tai L, Ekert PG, Huang DC, Norris F, Lindemann RK, Johnstone RW, Dixit VM, Strasser A. The $\mathrm{BH} 3$-only protein bid is dispensable for DNA damage- and replicative stress-induced apoptosis or cell-cycle arrest. Cell. 2007; 129:423-433.

40. Kang SG, Chung H, Yoo YD, Lee JG, Choi YI, Yu YS. Mechanism of growth inhibitory effect of Mitomycin-C on cultured human retinal pigment epithelial cells: apoptosis and cell cycle arrest. Curr Eye Res. 2001; 22:174-181.

41. Kumaresan KR, Lambert MW. Fanconi anemia, complementation group A, cells are defective in ability to produce incisions at sites of psoralen interstrand cross-links. Carcinogenesis. 2000; 21:741-751.

42. Kumaresan KR, Hwang M, Thelen MP, Lambert MW. Contribution of XPF functional domains to the 5' and $3^{\prime}$ incisions produced at the site of a psoralen interstrand crosslink. Biochemistry. 2002; 41:890-896.

43. Zhang P, Sridharan D, Lambert MW. Nuclear $\alpha$ spectrin differentially affects monoubiquitinated versus nonubiquitinated FANCD2 function after DNA interstrand cross-link damage. J Cell Biochem. 2016; 671-683. 